\title{
Mejoramiento de la Tenacidad de Polipropileno con nanoparticulas de Carbonato de Calcio
}

\author{
Percival Soni $^{1}$, Estrella Ruiz ${ }^{1}$,Tomas Lozano ${ }^{* 1}$, Ana Beatriz Morales ${ }^{1}$, Pierre Lafleur ${ }^{2}$, Guillermo \\ Martinez $^{3}$, Saul Sanchez ${ }^{3}$, Rodolfo Barragan Ramirez ${ }^{4}$ \\ ${ }^{1}$ Instituto Tecnológico de Cd. Madero, División de Estudios de Posgrado e Investigación, Juventino Rosas y Jesús Urueta, Col. Los \\ Mangos, C.P. 89440, Cd. Madero, Tamaulipas, México \\ ${ }^{2}$ École Polytechnique de Montréal, Chemical Engineering Department. P.O Box 6079, Stn Centre-Ville. Montreal, Quebec H3C $3 A 7$, \\ Canada. \\ ${ }^{3}$ Centro de Investigación en Química Aplicada (CIQA). Blvd. Enrique Reyna No. 140, C.P. 25100, Saltillo, Coahuila, México \\ ${ }^{4}$ Facultad de Ingeniería, Universidad Autónoma de Tamaulipas, Matamoros s/n, Zona Centro, 870000, Cd. Victoria, Tamaulipas, \\ Mexico.
}

Recibido $X X X X X X X$ 20XX, Aceptado $X X X X X X X 20 X X$

\section{Resumen}

Las propiedades mecánicas de nanocompuestos de polipropileno (PP) con carbonato de calcio $\left(\mathrm{CaCO}_{3}\right)$ fueron estudiados en este trabajo. Se utilizó ácido esteárico como un nuevo modificador de interfaz durante la preparación de los nanocompuestos utilizando una extrusora de doble husillo. La concentración en peso del nanorelleno $\left(\mathrm{CaCO}_{3}\right)$ en los compuestos fue de $1,5 \%, 2,5 \%$ y 5,0 \%, respectivamente. Se hicieron caracterizaciones a los nanocompuestos de resistencia al impacto y ensayos de tracción. La interacción entre $\mathrm{CaCO}_{3}$ y ácido esteárico se analizó mediante FTIR. Los espectros de infrarrojo no mostraron ninguna reacción química entre $\mathrm{CaCO}_{3}$ y ácido esteárico. El alargamiento a la rotura y la resistencia al impacto se incrementaron en los nanocompuestos utilizando un modificador de interfaz (ácido esteárico en la tolva de la extrusora).

Palabras clave: propiedades mecánicas, nanocompuestos, acido esteárico, caracterizaciones.

\section{Abstract}

The mechanical properties of polypropylene (PP)-calcium carbonate $\left(\mathrm{CaCO}_{3}\right)$ nanocomposites were studied in this work. In this study stearic acid was used as a new interface modifier during the compounding of the nanocomposites with a twin screw extruder. The concentration in weight of the nanofiller in the compounds was of $1.5 \%, 2.5 \%$ and $5.0 \%$ respectively. The nanocomposites were characterized by impact resistance and tensile tests. The interaction between $\mathrm{CaCO}_{3}$ and stearic acid was analyzed by FTIR. The infrared spectra did not show any chemical reaction between $\mathrm{CaCO}_{3}$ and stearic acid. The elongation at break and impact resistance were increased in nanocomposites with interface modifier (stearic acid in hopper of the extruder).

Keywords: mechanical properties, nanocomposites, stearic acid, characterized.

\section{Introducción}

Investigaciones de mezclas polímero-polímero así como mezclas polímero con nanoparticulas en los últimos años han tenido un gran auge, debido a la sinergia favorable en cuanto al mejoramiento de las propiedades mecánicas y resistencia térmica de los materiales plásticos de partida, resultando en un nuevo material [8].

Esta combinación de componentes se realiza normalmente al estado fundido por un proceso de extrusión. Este gran interés actual de mezclado de materiales es de que solo un equipo como un extrusor es necesario para la obtención de un nuevo material plástico con propiedades importantes, en cambio la síntesis de un nuevo material polimérico con propiedades especificas resulta extremadamente costoso $[1,2]$.

De igual manera el polipropileno es un material con mayor opción en el futuro, este hecho se ve justificado con el habito creciente de su mercado, considerándolo uno de los más atractivos por las ventajosas condiciones de competividad económica que caracterizan al polipropileno como miembro del grupo de los termoplásticos de gran consumo frente a los ingenieriles. La amplia gama de propiedades del polipropileno, lo hace adecuado para una gran variabilidad de aplicaciones en diferentes sectores, y marca pauta ante los materiales del futuro, además de suponer una alternativa, mucho más económica [4].

Las propiedades de polímeros reforzados con partículas minerales dependen significativamente del estado de dispersión en la matriz plástica. Los materiales con una distribución muy grande de partículas conducirán a propiedades mecánicas muy pobres. En particular, aglomerados con dimensión importante jugarán el papel de defectos e iniciarán fracturas, reduciendo considerablemente el desempeño mecánico del polímero reforzado [8].

La adición del carbonato de calcio genera un producto con un excelente equilibrio entre la rigidez y el impacto, además, de poder aumentar la dureza de las resinas. Entre los rellenos, éstos proporcionan una apariencia superior y consistencia del color.

Cabe mencionar que el ácido esteárico no ha sido reportado en la literatura como modificador interfacial entre el polímero y las nanopartículas de carbonato de 
calcio. Una ventaja importante del ácido esteárico es su bajo costo y encontrado fácilmente en el mercado.

Este pacta con materiales plásticos con mejores propiedades mecánicas con una nueva propuesta de modificación de nanopartículas de carbonato de calcio con ácido esteárico y también este último actuando como modificador interfacial entre el polímero y las nanopartículas.

\section{Parte experimental}

\subsection{Materiales y preparación de las muestras}

Los materiales usados en este estudio fue el polipropileno proporcionado por Indelpro, S.A. de C.V. ubicada en el Puerto Industrial en Altamira, Tamaulipas México. Este PP es un Profax (Pellet en gránulo translúcido, obtenido por extrusión) conocido en el mercado como XH1760. El ácido esteárico $\left(\mathrm{C}_{18} \mathrm{H}_{36} \mathrm{O}_{2}\right)$ utilizado fue comprado a la empresa Sigma-Aldrich (95\% de pureza) y fue utilizado como agente acoplante entre carbonato de calcio $\left(\mathrm{CaCO}_{3}\right)$ y el PP.

Las nanopartículas de carbonato de calcio con un tamaño de partícula de 10 a 80 nanómetros (nm), en forma de polvo blanco, fue comprado a la empresa American Elements (98\% de pureza).

Para la preparación de los nanocompuestos, se varió la cantidad de carbonato de calcio en 3 diferentes concentraciones (ver Tabla 1).

Carbonato de calcio modificado fue preparado utilizando el siguiente procedimiento con acido esteárico propúesto por Tabtiang and Venables ${ }^{16}$ and Li et al. ${ }^{17}$.

$\mathrm{E} 1 \mathrm{CaCO}_{3}$ se secó a $80^{\circ} \mathrm{C}$ durante 24 horas. El CaCO3 seco se mezcla con 1,5 partes por cien de ácido esteárico en un mezclador Henschel. La mezcla se inició a baja velocidad de $1,400 \mathrm{rpm}$ durante $5 \mathrm{~min}$ y luego aumentó a la alta velocidad de $2.800 \mathrm{rpm}$ durante $15 \mathrm{~min}$ y la temperatura en esta velocidad de la mezcla alcanzó el $43{ }^{\circ} \mathrm{C}$. Finalmente, la cámara se enfrió a $30^{\circ} \mathrm{C}$ a baja velocidad. $\mathrm{CaCO} 3$ con tratamiento de superficie se almacenó a temperatura ambiente en desecadores.

Un extrusor doblehusillo Werner and Pfleiderer se utilizó para prepara los nanocompuestos. La velocidad del tornillo fu de $50 \mathrm{rpm}$ y el perfil de temperatura fue de $175^{\circ} \mathrm{C}$ en la sección de alimentación a $190^{\circ} \mathrm{C}$ en el dado. El gasto másico del extrusor fue de $10 \mathrm{~kg} / \mathrm{hr}$.

Tres clases de composición fueron llevados a cabo de la siguiente manera:

1. $\mathrm{CaCO}_{3}$ sin modificar y PP fueron introducidos en la tolva del extrusor (bloque 1 de nanocompuestos).

2. $\mathrm{CaCO}_{3}$ modificado y PP fueron incorporados en la tolva del extrusor (bloque 2 nanocomuestos).

3. $\mathrm{CaCO}_{3}$, PP y $2 \%$ en peso de acido estearico por peso total de compuesto fueron introducidos en la tolva del extrusor.

Tabla 1. Muestras preparadas

\begin{tabular}{|l|l|l|l|}
\hline & Bloque 1 & Bloque 2 & Bloque 3 \\
\hline Muestras & PP/UnC1.5 & PP/MnC1.5 & PP/MTnC1.5 \\
\hline Muestras & PP/UnC2.5 & PP/MnC2.5 & PP/MTnC2.5 \\
\hline Muestras & PP/UnC5 & PP/MnC5 & PP/MTnC5 \\
\hline
\end{tabular}

En donde el bloque 1 representa a las mezclas de carbonato de calcio sin tratar y el bloque 2 las mezclas de carbonato de calcio con tratamiento de acido esteárico. Ls muestras del bloque 3 consiten con $\mathrm{CacCO} 3$ tratado y con cantidades extras de $\mathrm{CaCO} 3$ introducidas en la tolva del extruor.

Las diferentes concentraciones ocupadas de carbonato de calcio se muestran en partes por cien (ppc) de PP

\subsection{Ensayos de Impacto}

Ensayos de impacto Izod se evaluaron con metodo ASTM D256

\subsection{Ensayo de tracción}

Para el estudio de los nanocompuestos y polipropileno puro, se utilizó una maquina universal marca Shimadzu AGS-20kNG (ver figura 1). La velocidad utilizada para cada prueba fue de $10 \mathrm{~mm} / \mathrm{min}$.

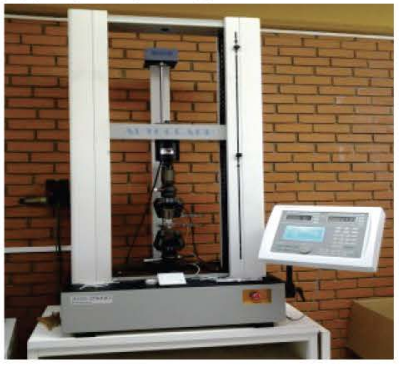

Figura 1. Maquina Universal

Las pruebas y las probetas utilizadas, se fundamentan con la norma ASTM D-638-10 para propiedades de tensión de plásticos. Se utilizaron 3 probetas por cada nanocompuesto , las cuales se fabricaron con un molde por el método de inyección, y de los tres ensayos realizados para cada nanocompuesto se obtiene una media aritmética de los resultados obtenidos.

\subsection{Infrarrojo (FTIR)}

Se utilizó un FTIR de marca Perkin Elmer Spectrum 100 equipado con un ATR de marca PIKE con punta de diamante (ver figura 2). El programa que se uso para procesar los datos fue el Spectrum. Se usó para analizar alguna posible reacción debido a la incorporación del ácido esteárico a los nanocompuestos. Por esta razón se analizó la matriz polimérica (XH1760 Profax) y el ácido esteárico para poder comparar los espectros. El número de onda utilizada fue de 4000 a $600 \mathrm{~cm}^{-1}$ en la región del infrarrojo medio, graficando contra \% de transmitancia para hacer los espectros. Para este análisis, se tomó una pequeña muestra de cada material (nanocompuestos).

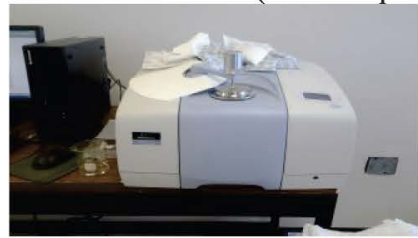

Figura 2. FTIR equipado con ATR 


\section{Resultados y Discusión}

\subsection{Espectroscopía en el infrarrojo (FTIR)}

La Figura 3 muestra los espectros IR del ácido esteárico, nanocarbonato no modificado y modificado. En la figura 3a se observa un pico de alta intensidad a $1700 \mathrm{~cm}^{-1}$ debida a la flexión del grupo $\mathrm{C}=\mathrm{O}$, la señal a $1430 \mathrm{~cm}^{-1}$ corresponde a la flexión del grupo O-H, y el pico a $1295 \mathrm{~cm}^{-1}$ corresponde a la flexión del grupo C-O, éstos picos característicos comprueban la existencia del grupo- $\mathrm{COOH}$.

La figura $3 \mathrm{~b}$ muestra el espectro IR del nanocarbonato no modificado, en el cual se resaltan las señales características del ión $\mathrm{CO}_{3}{ }^{++}$en $1400 \mathrm{~cm}^{-1}$ y $810 \mathrm{~cm}^{-1}$, asimismo en $710 \mathrm{~cm}^{-1}$ se observa la señal característica de la calcita (carbonato de calcio). La figura $3 \mathrm{c}$ representa el espectro del nanocarbonato modificado con ácido esteárico, donde se observan las mismas señales características del carbonato de calcio a 1400 y $810 \mathrm{~cm}^{-1}$ para el ión de carbonato y a $710 \mathrm{~cm}^{-1}$ para la calcita. En esta última imagen no se encuentra señal alguna del grupo carbonilo correspondiente al ácido esteárico, por lo cual se puede concluir que no existe reacción química alguna entre el carbonato de calcio y el ácido esteárico, sino que solo hay una interacción física.

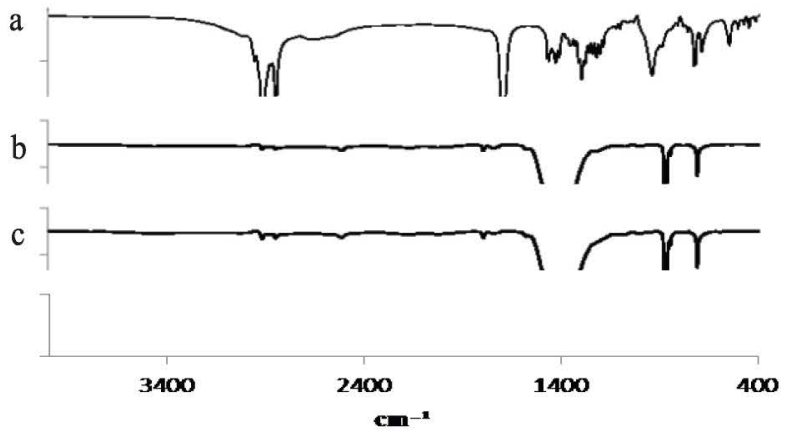

Figura 3. Espectros de Infrarrojo de nanomateriales

\subsection{Ensayo de Impacto}

En la Tabla 2 se muestran los resultados de resistencia al impacto de los nanocompuestos preparados. Se observa que para los compuestos con mineral no tratado (bloque 1) el valor es menor al del PP sin carga $\left(1.28 \mathrm{KJ} / \mathrm{m}^{2}\right), y$ que para los compuestos con nano carbonato modificado con ácido esteárico (bloque 2) tampoco se obtienen valores mayores al de la resina sin carga, por el contrario, la resistencia al impacto en este bloque de compuestos disminuye apreciablemente. Para los compuestos con relleno modificado y ácido esteárico extra agregado en la tolva (bloque3) la resistencia al impacto aumentó significativamente obteniendo resultados mayores a 1.5 $\mathrm{KJ} / \mathrm{m}^{2}$, esto indica que el ácido esteárico está influyendo como agente interfacial, logrando que las nanopartículas se dispersen mejor dentro de la matriz polimérica que a su vez distribuyen mejor la energía de impacto logrando así una mejor resistencia mecánica.

Tabla 2. Propiedades de Impacto

\begin{tabular}{|c|c|}
\hline Parámetro & $\begin{array}{c}\text { Resistencia al } \\
\text { impacto }\end{array}$ \\
\hline Unidades & $\mathrm{KJ} / \mathrm{m} 2$ \\
\hline Muestra & \\
\hline$P P$ & 1.28 \\
\hline$P P / U n C 1.5$ & 1.19 \\
\hline$P P / U n C 2.5$ & 1.66 \\
\hline$P P / U n C 5$ & \\
\hline$P P / M n C 1.5$ & \\
\hline$P P / M n C 2.5$ & 1.14 \\
\hline$P P / M n C 5$ & 1.03 \\
\hline$P P / M T n C 1.5$ & 1.51 \\
\hline$P P / M T n C 2.5$ & 1.5 \\
\hline$P P / M T n C 5$ & 1.58 \\
\hline
\end{tabular}

\subsection{Pruebas de tracción}

La Tabla 3 muestra los resultados de los análisis de tracción para los compuestos preparados. Se observa que el módulo de elasticidad aumentó ligeramente para los compuestos con relleno no modificado y aumentó significativamente para los compuestos con nanocarbonato modificado con ácido esteárico, lográndose un aumento del 8 y el $13 \%$ para los compuestos $\mathrm{PP} / \mathrm{MnC} 2.5$ y $\mathrm{PP} / \mathrm{MnC} 5$ respectivamente. Por el contrario para los compuestos con relleno modificado y ácido esteárico extra agregado en tolva el valor de esta propiedad disminuyó ligeramente en comparación con el valor para el PP sin relleno. Estos resultados van en concordancia con el comportamiento de la elongación de los compuestos, ya que para el bloque de compuestos con nanocarbonato no tratado (bloque 1) la máxima elongación fue baja comparada con la del PP, en el bloque de compuestos con relleno modificado (bloque 2) la elongación mostró un ligero aumento, obteniéndose valores entre un 4 y un $8 \%$ mayores con respecto de la elongación máxima del PP sin relleno, mientras que para los compuestos con nanocarbonato y ácido esteárico en tolva (bloque3) la máxima elongación fue la más alta en comparación con la de los demás compuestos y con el PP sin relleno, obteniendo valores hasta del $23 \%$ de aumento para los compuestos con una concentración de nanocarbonato de $5 \%$. Este comportamiento mecánico es difícil de conseguir puesto que las partículas de tamaño nanométrico son difíciles de dispersar en una matriz polimérica, y con ello la dificultad de lograr mejorías en propiedades como la elongación, sin embargo, con la introducción de un modificador interfacial como lo es el ácido esteárico, las interacciones físicas débiles entre polímero-relleno se favorecen generando un mejor mojado de las nanoparticulas por el polímero y asi a una 
mejor dispersión del relleno, al haber una mejor dispersión se limita o elimina la formación de aglomeraciones, causantes principales de la ruptura de un material en pruebas de tracción e impacto. Normalmente la elongación en la ruptura se ve reducida en nanocompuestos con matrice polimérica $[13,14,15]$. Es difícil de encontrar en literatura aumentos en elongación de la matrice con nanoparticulas. La adición de nanoparticulas reduce ductilidad de la matrice polimérica.

Tabla 3. Propiedades mecanicas de los compuestos

$\mathrm{PP} /$ Nanocarbonato

\begin{tabular}{|c|c|c|c|c|}
\hline Parametro & Modulo & $\begin{array}{c}\text { Resistenci } \\
\text { a a la } \\
\text { tension }\end{array}$ & $\begin{array}{c}\text { Maxima } \\
\text { elongacio } \\
n\end{array}$ & $\begin{array}{c}\text { Resistenci } \\
\text { a al } \\
\text { impacto }\end{array}$ \\
\hline Unidades & Mpa & Mpa & $\%$ & $K J / m 2$ \\
\hline Muestra & & & & \\
\hline PP & 1214.17 & 48.6111 & 849.082 & 1.28 \\
\hline PP/UnC1.5 & 1495.47 & 44.1667 & 831.532 & 1.19 \\
\hline PP/UnC2.5 & 1204.51 & 45.8333 & 799.043 & 1.66 \\
\hline PP/UnC5 & & 47.2222 & 827.822 & \\
\hline PP/MnC1.5 & & 51.3889 & 883.853 & \\
\hline PP/MnC2.5 & 1322.24 & 49.7222 & 895.89 & 1.14 \\
\hline PP/MnC5 & 1373.26 & 51.1111 & 933.85 & 1.03 \\
\hline PP/MTnC1.5 & 1131.95 & 48.8889 & 975.162 & 1.51 \\
\hline PP/MTnC2.5 & 1014.39 & 51.1111 & 1017.47 & 1.5 \\
\hline PP/MTnC5 & 1204.51 & 51.1111 & 1046.41 & 1.58 \\
\hline & & & & \\
\hline
\end{tabular}

\section{Conclusiones}

En los espectros de infrarrojo se mostro que no existe ningún tipo de reacción química entre el acido esteárico y el carbonato de calico, las interacciones son principalmente fisicas.

El acido esteárico redujo la aglomeración del nanorelleno y llevó a una mejor humectación de las partículas minerales por el PP, debido a las interacciones entre el $\mathrm{CaCO}_{3}$ y el PP

La adición de ácido esteárico como modificador interfacial mejoró las propiedades de tenacidad del PP cargado con $\mathrm{CaCO}_{3}$ (resistencia al impacto y la elongación en la rotura suoeriores). Estas mejoras significativas de tenacidad son difíciles de encontrar en los publicados en la literatura, en la mayoría de los casos se reducen estas propiedades con la adición de un nanorelleno a un polímero.

\section{Referencias}

1. Rosas, A., Gonzales Madariaga, F., Proceso de Extrusión, disponible en línea: http://www.slideshare.net/betorossa/proceso-de-extrusin-deplsticos, abril 2011.

2. López, J., Extrusión, disponible en línea:

http://www.slideshare.net/JorgeLopez87/extrusora, abril 2012.

3. ANiQ, Clasificación de los plásticos, disponible en línea: http://www.aniq.org.mx/cipres/clasificacion.asp, septiembre 2009

4. Mariano R., Polipropileno, disponible en línea:

http://tecnologiadelosplasticos.blogspot.mx/2011/06/polipropile no.html, junio 2011

5. Morales J., La maquina de inyección, disponible en: http://docencia.udea.edu.co/ingenieria/moldes inyeccion/unidad 2/maquina.html, marzo 2009

6. Téllez Salero S., Introducción al proceso de inyección de plásticos. Disponible en línea:

http://www.cnad.edu.mx/sitio/matdidac/md/plasticos/MANUAL PARTINYPLAS.pdf, junio 2010

7. Rigail Cedeño A., Mendoza Rumiguano A., Evaluación de compuestos de polipropileno y carbonato de calcio para aplicaciones industriales. Disponible en línea:

http://www.dspace.espol.edu.ec/bitstream/123456789/17027/1/ Articulo\%20de\%20Tesis\%20de\%20Grado.pdf, Mayo 2010.

8. Moral Borque, A., Nogueira López M., Materiales de última generación y materiales eficientes: Materiales compuestoscomposites. Disponible en línea:

https://portal.uah.es/portal/page/portal/GP EPD/PG-MAASIG/PG-ASIG-

200637/TAB42359/Introducci\%F3n\%20Composites\%20(Albert \% \% 20Moral\%20\%2B\%20Marar\%20Nogueira).pdf, junio 2007

9. Seymour R., Carraher C., Introducción a la química de los polímeros, Editorial Reverté, D.F., México, 1995

10. Rodríguez J., Ensayo de tracción universal. Disponible en línea: http://ocw.uc3m.es/ciencia-e-oin/tecnologia-demateriales-industriales/practicas-1/Practica II-TRACCION.pdf, noviembre, 2006

11. Gómez R., Murillo R., Espectroscopia infrarroja. Disponible en línea:

http://sistemas.fciencias.unam.mx/ fam/Infrarroja.pdf, Mayo, 2007.

12. Montalvo Arenas C., Microscopia. Disponible en línea: http://www.facmed.unam.mx/deptos/biocetis/PDF/Portal\%20de $\% 20$ Recursos $\% 20$ en $\% 20$ Linea/Apuntes $/ 2$ microscopia.pdf, Agosto, 2010

13. Pavlidou S, Papaspyrides CD, ProgPolymSci., 33(2008) 1119.

14. Ray S and Okamoto M,ProgPolymSci., 28(2003)1539

15. Sharma K, Nayak SK,Polym Degradation and Stability, 94 (2009)132.

16. Tabtiang A and Venables R,Compos Interface,6 (1999) 65.

17. Li Z, Shen SY, Peng JR, Yang CR, Key Eng Mat., 249 (2003)409 